\title{
Expression of a Mutant Human Fibrillin Allele upon a Normal Human or Murine Genetic Background Recapitulates a Marfan Cellular Phenotype
}

\author{
Zayd A. Eldadah, * Thomas Brenn, ${ }^{\star}$ Heinz Furthmayr, ${ }^{\star}$ and Harry C. Dietz \\ ${ }^{*}$ Center for Medical Genetics, The Johns Hopkins University School of Medicine, Baltimore, Maryland 21205; and ${ }^{\ddagger}$ Department of \\ Pathology, Stanford University Medical Center, Stanford, California 94305
}

\begin{abstract}
The Marfan syndrome (MFS) is a connective tissue disorder inherited as an autosomal dominant trait and caused by mutations in the gene encoding fibrillin, a 350-kD glycoprotein that multimerizes to form extracellular microfibrils. It has been unclear whether disease results from a relative deficiency of wild-type fibrillin; from a dominant-negative effect, in which mutant fibrillin monomers disrupt the function of the wild-type protein encoded by the normal allele; or from a dynamic and variable interplay between these two pathogenetic mechanisms. We have now addressed this issue in a cell culture system. A mutant fibrillin allele from a patient with severe MFS was expressed in normal human and murine fibroblasts by stable transfection. Immunohistochemical analysis of the resultant cell lines revealed markedly diminished fibrillin déposition and disorganized microfibrillar architecture. Pulse-chase studies demonstrated normal levels of fibrillin synthesis but substantially reduced deposition into the extracellular matrix. These data illustrate that expression of a mutant fibrillin allele, on a background of two normal alleles, is sufficient to disrupt normal microfibrillar assembly and reproduce the MFS cellular phenotype. This underscores the importance of the fibrillin amino-terminus in normal microfibrillar assembly and suggests that expression of the human extreme 5 ' fibrillin coding sequence may be sufficient, in isolation, to produce an animal model of MFS. Lastly, this substantiation of a dominant-negative effect offers mutant allele knockout as a potential strategy for gene therapy. (J. Clin. Invest. 1995. 95:874-880.) Key words: Marfan syndrome - dominantnegative mutation • microfibril • extracellular matrix
\end{abstract}

\section{Introduction}

The Marfan Syndrome (MFS) ${ }^{1}$ is a systemic disorder of connective tissue with autosomal dominant inheritance and a preva-

Address correspondence to Harry C. Dietz, MD, The Johns Hopkins University School of Medicine, Ross 1170, Baltimore, MD 21205. Phone: (410) 614-0729; FAX: (410) 614-2256.

Received for publication 10 August 1994 and in revised form 8 November 1994.

1. Abbreviation used in this paper: MFS, Marfan Syndrome.

J. Clin. Invest.

(C) The American Society for Clinical Investigation, Inc.

$0021-9738 / 95 / 02 / 0874 / 07 \quad \$ 2.00$

Volume 95, February 1995, 874-880 lence of about 1 in 10,000 individuals. Hallmark features of the disease include ocular impairment, principally lens dislocation and myopia; skeletal abnormalities such as dolichostenomelia and joint laxity; and, most significantly, cardiovascular pathology, including aortic root dilatation and dissection, mitral valve prolapse, and mitral and aortic valvular insufficiency (1-3). Positional cloning and candidate gene analyses recently culminated with the identification of FBN1, the gene encoding fibrillin, as the site of most, if not all, MFS-producing mutations (4-6).

Fibrillin is a $350-\mathrm{kD}$ glycoprotein and the principal component of the extracellular microfibril (7). It is abundantly expressed in many tissues altered in the Marfan phenotype, including the skin, vascular wall, cartilage, suspensory ligament of the lens, and alveolar wall (8). The structure of the protein is complex and redundant. It is comprised of 46 EGF-like motifs which are tandemly repeated and irregularly interspersed with TGF- $\beta_{1}$ binding protein (TGF- $\beta_{1}$ bp)-like domains. A number of apparently unique cysteine-rich repeats are also present $(9$, 10 ). The abundance of cysteine-rich EGF-like and TGF- $\beta_{1}$ bplike domains enables fibrillin monomers to participate in intramolecular as well as intermolecular interactions. Indeed, upon secretion into the extracellular matrix, fibrillin monomers rapidly form disulfide-bonded aggregates, which, in conjunction with other matrix components, lead to the formation of mature microfibrils $(11,12)$. Rotary shadowing of these multimeric units reveals a beaded-string appearance, and it has been proposed that fibrillin monomers arrange in a head-to-tail fashion $(8,13)$. Mature microfibrils have an average diameter of 10 $\mathrm{nm}$, and the "beads" have been shown to be spaced at regular intervals of 26 to $60 \mathrm{~nm}$ (14).

Several lines of evidence suggest that MFS follows a dominant-negative mechanism of pathogenesis. The disorder is inherited in an autosomal dominant fashion and is caused by mutations in a gene encoding a protein known to multimerize. In addition, a hallmark feature of classic MFS is severe deficiency of extracellular fibrillin, far below the amount predicted by a single functioning FBN1 allele $(15,16)$. Finally, mutations causing very reduced levels of FBN1 mutant transcript have been associated with an extremely mild disease phenotype (17). A dominant-negative scenario would thus involve mutant fibrillin monomers disrupting normal fibrillin multimer formation and consequently producing disease.

At the cellular level, fibroblasts cultured from MFS patients have been shown to exhibit consistent and relatively specific abnormalities by immunohistochemical and pulse-chase analyses. Indirect immunofluorescence of normal human fibroblasts with monoclonal antibodies to fibrillin results in a highly reproducible and distinct staining pattern. An abundant, organized meshwork of microfibrils is observed. In contrast, fibroblasts 
Table I. Oligonucleotide Sequences

\begin{tabular}{|c|c|}
\hline Oligonucleotide & Sequence \\
\hline F5UT-1S: & $5^{\prime}-$ GCA AGA GGC GGC GGG AG $-3^{\prime}$ \\
\hline FB1S: & 5'-GGA TTT ACC GTG CTT TTA GC-3' \\
\hline FB1CS: & $5^{\prime}$-AAC GTG AAG GAA ACC AGA G-3' \\
\hline FB1AS: & $5^{\prime}-$ TTC TGG CAT AGA CAG TGA TC-3' \\
\hline FB2AS: & $5^{\prime}$-GGC CTG GCA TTC ATC CAC-3' \\
\hline ME1S-1A: & $5^{\prime}$-ACG AGC CAT GGG GCG GAC-3' \\
\hline ME1S-B: & $5^{\prime}$ - CGG ACG CCA ATT TGG AGG C-3' \\
\hline ME1S-C: & $5^{\prime}$ - CGG GCC AAG AGA AGA GGC-3' \\
\hline ME3AS-A & $5^{\prime}-$ CTG GAG CCA CAG GAA GGA G-3' \\
\hline ME3AS-B & $5^{\prime}$ - CAA GTG CAC ATA TTT GGC CTC- $3^{\prime}$ \\
\hline SP6: & 5'-ATT TAG GTG ACA CTA TAG-3' \\
\hline T7: & 5'-ATT ACG ACT CAC TAT AG-3' \\
\hline
\end{tabular}

from MFS patients generally exhibit a striking reduction in staining, with the microfibrillar lattice showing only occasional, scattered, and wispy fibers (18). These immunohistochemical profiles can largely be grouped into two categories: $(a)$ marked decrease in deposition, and $(b)$ variable decrease in deposition with characteristic abnormalities of microfibrillar architecture. Pulse-chase analysis of radiolabelled fibrillin has been used to identify and quantify defects in fibrillin synthesis, secretion, and incorporation into the extracellular matrix $(12,15)$. The method has enabled five apparently distinct groups of MFS patients to be categorized based on their fibrillin synthesis and deposition profiles (16). For example, cells carrying FBN1 missense mutations consistently show near normal levels of fibrillin synthesis but markedly diminished matrix deposition. Cells from patients with reduced levels of mutant transcript demonstrate $\sim 50 \%$ fibrillin synthesis and variable deposition into the matrix (16). As predicted by a dominant-negative pathogenesis, patients with mild disease show near complete deposition of synthesized fibrillin while those with a severe phenotype show much reduced $(6-25 \%)$ deposition (16).

A patient identified with FBN1 mutation $247 \mathrm{G}^{+1}$ to $\mathrm{A}$, a splice site alteration producing classic and severe MFS, was the basis for this study. The splice site change leads to skipping of FBN1 exon 2, a frameshift, and a consequent premature termination codon in exon 4 . To directly address the issue of a dominant-negative pathogenesis, we assembled an expression construct containing this del exon 2 allele and stably transfected normal human and murine fibroblasts. Immunohistochemical analysis of transfected cell lines revealed markedly diminished fibrillin deposition and disorganized microfibrillar architecture. Pulse-chase studies of these cells demonstrated normal levels of fibrillin synthesis but substantially reduced deposition of fibrillin into the extracellular matrix.

\section{Methods}

Subcloning of del exon 2 mutant cDNA. Total RNA was extracted with RNAzol per the manufacturer's protocol (Tel-Test, Friendswood, TX) from a primary fibroblast culture generated by skin biopsy of patient MS-8, who harbors mutation del exon 2 . Approximately $5 \mu \mathrm{g}$ of total RNA was reverse transcribed as described (5), and the resultant CDNA was amplified by the PCR using oligonucleotides F5UT-1S and FB2AS with a PCR reagent kit (GeneAmp; Perkin-Elmer Cetus, Norwalk, CT). Sequences of all oligonucleotides used in this study are listed in Table I. PCR conditions were $95^{\circ} \mathrm{C} \times 3$ min followed by 30 cycles of $95^{\circ} \mathrm{C}$
pDEL2 construct

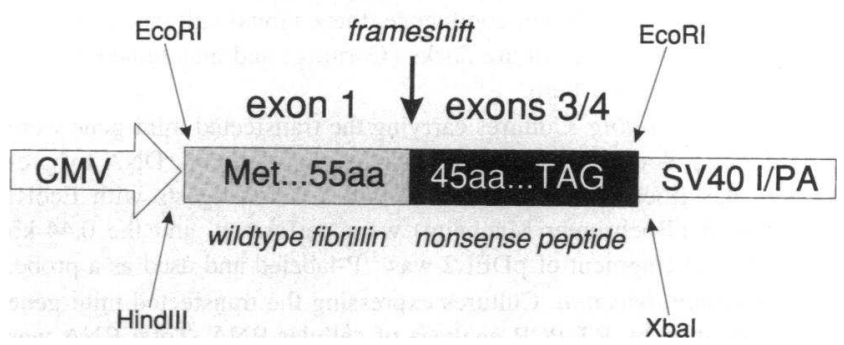

Figure 1. Schematic of the experimental expression construct pDEL2 showing the composition of the mutant polypeptide predicted by the del exon 2 allele. The insert is preceded by a CMV promoter and followed by an SV40 intron and polyadenylation signal. Restriction sites are shown. The EcoRI sites are 345 bp apart, and the HindIII and XbaI sites are 439 bp apart.

$\times 30 \mathrm{~s}, 58^{\circ} \mathrm{C} \times 30 \mathrm{~s}, 72^{\circ} \mathrm{C} \times 30 \mathrm{~s}$ then one cycle of $72^{\circ} \mathrm{C} \times 5 \mathrm{~min} .1$ $\mu \mathrm{l}$ of this reaction product served as template for a semi-nested PCR using the same conditions but primers F5UT-1S and FB1AS. Following the manufacturer's protocol for the TA Cloning Kit (Invitrogen, San Diego, CA), this secondary PCR product was subcloned into the PCRII vector. A recombinant plasmid containing the 330 -bp insert was identified and characterized by direct sequencing using a dsDNA Cycle Sequencing Kit (GIBCO BRL, Gibco Gaithersburg, MD), thus confirming the identity and orientation of the insert.

Preparation of expression construct. The insert was released from pCRII by digestion with HindIII and XbaI (Boehringer Manheim Corp., Indianapolis, IN) and subcloned into the HindIII and XbaI sites in pcDNA3, a eukaryotic expression vector containing a CMV promoter and neomycin and ampicillin resistance genes (Invitrogen). Insert orientation and identity in the final expression construct (designated pDEL2) were confirmed by cycle sequencing as above. A schematic of this construct is shown in Fig. 1. The predicted protein consists of 100 amino acids from the amino terminus of fibrillin with the latter 45 encoded by out-of-frame codons. Plasmid preparations of pDEL2 as well as intact pcDNA3 (to serve as a control) were made using the Plasmid Maxi Kit and supplied protocol (QIAGEN Inc., Chatsworth, CA).

Transfection of cultured fibroblasts. Control human and mouse NIH 3T3 (American Type Culture Collection, Rockville, MD) fibroblasts were grown on 100-mm tissue culture dishes (Corning, Corning, NY) with Eagle's Minimal Essential Medium plus $10 \%$ fetal bovine serum and $1 \times$ penicillin-streptomycin solution (GIBCO BRL). Cells were transfected when they reached $60-80 \%$ confluence. $70 \mu 1$ of DOTAP transfection reagent (Boehringer Manheim Corp.) were added to 180 $\mu$ l of HBS solution ( $20 \mathrm{mM}$ Hepes, $150 \mathrm{mM} \mathrm{NaCl}, \mathrm{pH} \mathrm{7.4)}$ ) in a sterile tube. $15 \mu \mathrm{g}$ of Qiagen-extracted plasmid DNA were added, and the solutions were mixed and allowed to incubate for $10 \mathrm{~min}$ at room temperature. $10 \mathrm{ml}$ of cell culture medium (composition as above) were then added to the transfection reagent-DNA mixture. The medium was aspirated from the fibroblast culture dishes, replaced with this $10 \mathrm{ml}$ of transfection solution, and incubated at $37^{\circ} \mathrm{C}$ with $5 \% \mathrm{CO}_{2}$ for $18 \mathrm{~h}$. Parallel transfections of pDEL2 (experimental) and pcDNA3 (control) were performed, and resultant cells from the experimental and control transfections were carried through all subsequent stages of antibiotic selection and phenotype analysis.

Clonal selection of transfected cells. The transfection solution was removed and replaced with fresh cell culture medium containing $\sim 35$ $\mu \mathrm{g} / \mathrm{ml}$ active neomycin (Genticin; GIBCO BRL). To remove dead and dying cells, cultures were rinsed daily with Hank's balanced salt solution (GIBCO BRL) and overlaid with fresh neomycin-containing culture medium. After $2 \mathrm{wk}$, isolated clonal colonies of $10-100$ cells could be observed. At this point the dishes were rinsed in Hank's balanced salt solution, and sterile $8 \times 8 \mathrm{~mm}$ cloning cylinders (Specialty Media, Lavallette, NJ) were used to isolate individual clonal colonies. After 
treatment with trypsin, the cells were harvested and transferred to single wells of a 24-well sterile tissue culture plate (Falcon; Becton Dickinson, Franklin Lakes, NJ). Upon confluence, these clonal cultures were then plated in $75-\mathrm{cm}^{2}$ tissue culture flasks (Corning) and maintained in neomycin-containing medium.

Southern blotting. Cultures carrying the transfected mini-gene were identified by Southern blot analysis of cellular genomic DNA by previously described methods (19). Genomic DNA digests with EcoRI and Bsu36I (Boehringer Manheim) were performed, and the 0.44-kb HindIII-Xbal fragment of pDEL2 was ${ }^{32} \mathrm{P}$-labeled and used as a probe.

Transcript detection. Cultures expressing the transfected mini-gene were identified by RT-PCR analysis of cellular RNA. Total RNA was extracted from each $75-\mathrm{cm}^{2}$ culture as above (5), and 5-10 $\mu \mathrm{g}$ were treated with $3 \mathrm{U}$ of RNase-free DNase I (Boehringer Manheim) for 30 min at $37^{\circ} \mathrm{C}$. After phenol-chloroform extraction, 1-5 $\mu \mathrm{g}$ of RNA were analyzed by RT-PCR following the manufacturer's protocol (RT-PCR Kit; Perkin-Elmer Cetus). Random hexamers and oligonucleotide FB1AS were used in the reverse-transcription reaction. To control for DNA contamination, the same amounts of this DNase-treated RNA were analyzed by PCR without prior reverse transcription. All products were electrophoresed through a $1 \%$ agarose gel and transferred to a nylon membrane using a Turboblotter apparatus as per the manufacturer's protocol (Schleicher \& Schuell, Inc., Keene, NH). Using a previously described allele-specific oligonucleotide hybridization method (20), the identity of the amplified cDNA was confirmed by hybridization with ${ }^{32} \mathrm{P}$-labeled oligonucleotide FB1S, which is complementary to sequence within FBN1 exon 1. For transcript quantification, RT-PCR was performed on DNase-treated total RNA that was extracted from representative mouse 3T3 transfected cell line E3. Random hexamers were used for reverse transcription. Each PCR reaction utilized sense and antisense primers that were exactly complementary to sequences in both mouse and human exons 1 and 3, respectively: ME1S-A and ME3AS-A; ME1S$A$ and ME3AS-B; ME1S-B and ME3AS-A. RT-PCR products were electrophoresed and Southern transferred as above and hybridized to radiolabeled oligonucleotide ME1S-C, which is complementary to both endogenous and mini-gene-derived transcripts, or oligonucleotide FB1CS, which is complementary only to the mini-gene transcript. Quantification was performed as previously described (17).

Immunohistochemistry. Single wells of a four-chamber slide (LabTek; Nunc, Naperville, IL) were seeded with $2 \times 10^{5}$ cells from each clonal culture and grown in neomycin-containing medium for $72 \mathrm{~h}$. The cells were rinsed with PBS and soaked in $-20^{\circ} \mathrm{C}$ acetone for $10 \mathrm{~min}$. The acetone was then replaced with fresh, room temperature PBS for 15 min. The PBS was removed, and the primary antibody (anti-fibrillin monoclonal 69, kindly provided by Dr. Lynn Sakai, Portland Shriners Hospital, Portland, Oregon) was added at 1:150 dilution in PBS. The antibody was incubated for $3 \mathrm{~h}$ at room temperature. The cells were then washed three times for 3 min each in PBS. The secondary antibody (FITC-conjugated anti-mouse IgG; Sigma Chem. Co., St. Louis, MO) at 1:256 dilution in PBS was then added to the cells for $30 \mathrm{~min}$ at room temperature. The cells were washed again three times for 3 min each in PBS, then stained for $5 \mathrm{~min}$ in $1 \mu \mathrm{g} / \mathrm{ml}$ propidium iodide (Sigma), and put through a final series of four 3-min PBS washes. The slides were fixed in mounting medium (Cytoseal 280; Stephens Scientific, Riverdale, NJ) and examined by fluorescent microscopy with a 450 490 -nm filter. Immunofluorescence was also performed on all cultures with a monoclonal antibody to fibronectin (Sigma), an extracellular matrix protein which is unaffected in MFS, to control for specificity.

Assessing presence of secreted polypeptide. Cells harboring the mini-gene construct as well as control cells harboring only the parent pcDNA3 vector were grown in chamber slides as described above but in cell culture medium without neomycin. After $24 \mathrm{~h}, 1 \mathrm{ml}$ of the medium from atop these cells was used to resuspend $2 \times 10^{5}$ normal, untransfected fibroblasts which had been pelleted by centrifugation. These cells were then plated in chamber slides and carried through the immunohistochemical procedure described above. If the mutant polypeptide were secreted into the medium of pDEL2-transfected cells, it would be expected to disrupt microfibrillogenesis.

Pulse-chase analysis. Pulse-chase analysis of radiolabeled fibrillin
Figure 2. Southern blots of $(A)$ EcoRI- and $(B)$ Bsu36I-digested genomic DNA using the HindIII-Xbal fragment of pDEL2 as a probe. The EcoRI digest demonstrates a $0.34-\mathrm{kb}$ restriction fragment specific to a representative clone $(E)$ of cells transfected with pDEL2. Signal is absent in a representive control cell line (C). The presence of the full-length $(5.8 \mathrm{~kb}) \mathrm{vec}$ tor is also seen and likely reflects incomplete digestion of the construct. In addition, hybridization with a $1.0-\mathrm{kb}$ band is seen in both lanes, reflecting cross-hybridization of the probe to the native fibrillin sequence present in both samples. The digest with Bsu36I, which cuts neither the insert nor parent vector, reveals a single band at 5.8 $\mathrm{kb}$ (the size of the vector + insert) in three representative clones (E2, E3, and E5), indicating stable replication of a nonintegrated expression plasmid. Again, signal is absent in the controls (C1, C2).

in these clonal cell cultures was performed by standard protocol without modification (15). Data for human and murine pDEL2-transfected cell lines were standardized to values obtained when the same parent lines were transfected with the insert-free expression vector alone.

\section{Results}

Identification of cultures harboring expression construct. Two Southern blots are shown in Fig. 2. The EcoRI digest (Fig. 2 $A$ ) demonstrates a $0.34-\mathrm{kb}$ restriction fragment that is specific to a representative clonal culture $(E)$ transfected with pDEL2. This conforms to the expected size of the mutant FBN1 allele excised from the expression construct with EcoRI. Signal is absent in the control (C). In addition, in the experimental as well as control sample there exists a $1.0-\mathrm{kb}$ EcoRI fragment, which reflects cross-hybridization of the probe with endogenous fibrillin sequence. The digest with Bsu36I (Fig. 2 B), which cuts neither the insert nor parent vector, reveals a single band at $5.8 \mathrm{~kb}$ (the size of intact pDEL2) in three representative clonal colonies of transfected 3T3 cells, indicating stable, episomal replication of the expression plasmid. Again, signal is absent in the controls $(\mathrm{C} 1, \mathrm{C} 2)$.

Identification of cultures expressing mutant transcript. The results of the RT-PCR-based assay are shown in Fig. $3 A$. The specific 295-bp band is seen in representative pDEL2- 


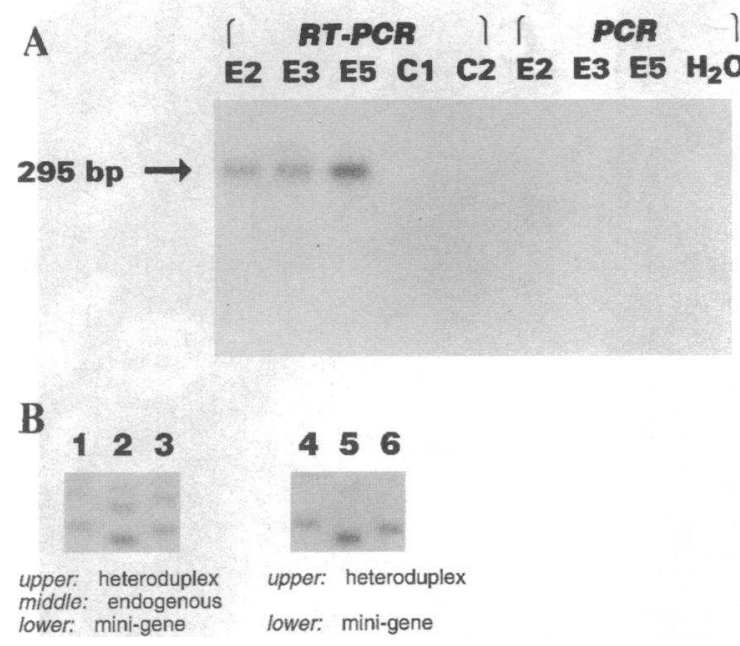

Figure 3. RT-PCR blots. (A) Transcript detection by RT-PCR is shown here for three representative clones of cells transfected with pDEL2 $(\mathrm{E} 2, \mathrm{E} 3, \mathrm{E} 5)$ and two controls $(C 1, C 2)$. DNase-treated cellular RNA was reverse transcribed and then amplified with construct-specific primers. Hybridization with an end-labeled oligonucleotide (FB1S) complementary to sequence within the 295-bp target is shown. Signal is absent in the two controls $(\mathrm{Cl}, \mathrm{C2})$ and in the same three experimental clones upon PCR of DNase-treated RNA without prior reverse transcription (a control for plasmid DNA contamination). (B) Quantification of transcript derived from the two endogenous mouse fibrillin alleles versus that derived from the mutant mini-gene in representative pDEL2transfected clone E3. Primers used for RT-PCR were completely complementary to both mouse and human fibrillin cDNA sequence. The smaller size of the pDEL2-derived RT-PCR products reflects skipping of $F B N 1$ exon 2 ( $83 \mathrm{bp}$ ). Lanes 1 and 4, primers ME1S-A and ME3AS$\mathrm{A}$, endogenous mouse-derived (EMD) product $=280 \mathrm{bp}$, mini-genederived (MD) product $=197 \mathrm{bp}$; lanes 2 and 5, primers ME1S-A and ME3AS-B, EMD product $=244 \mathrm{bp}, \mathrm{MD}$ product $=161 \mathrm{bp}$; lanes 3 and 6, primers ME1S-B and ME3AS-A, EMD product $=267 \mathrm{bp}$, MD product $=184 \mathrm{bp}$. RT-PCR products were Southern-transferred and hybridized to a labeled probe either complementary to both classes of transcript (ME1S-C) (lanes $1-3$ ) or specifically complementary to MD transcript (FB1CS) (lanes 4-6). The faint and largest band seen in each lane reflects slower migration of heteroduplex DNA.

transfected cultures E2, E3, and E5 and conforms to the expected PCR product size. Signal is absent from control clones $\mathrm{C} 1$ and $\mathrm{C} 2$ as well as from the experimental cultures carried through the control PCR (amplifying samples of DNase-treated RNA without prior reverse transcription). RT-PCR was also used to quantify the relative amounts of transcript derived from pDEL2 relative to that derived from the endogenous mouse alleles in representative transfected cell line E3 (Fig. $3 \mathrm{~B}$ ). On average, the contribution of pDEL2-derived transcript is $\sim 140 \%$ of that from the endogenous alleles. This represents an overestimate as the smaller, mini-gene-derived product would be preferentially amplified in a PCR-based assay. Nevertheless, these data suggest that the mutant mini-gene product is not grossly overproduced in this model system relative to the in vivo situation in patients with the Marfan syndrome.

Immunohistochemistry. Shown in Fig. $4(A-E)$ are photomicrographs of fibroblasts stained with a monoclonal antibody to fibrillin. Nuclei are stained with propidium iodide and appear orange. Microfibrils appear green. Normal human cells $(A)$ demonstrate the pattern of abundant, long, smooth, organized microfibrils that has been well characterized (18). A primary culture from patient MS-8 $(B)$, who harbors the mutation upon which this study was based, demonstrates reduced extracellular utilization of fibrillin and disorganized microfibrillar architecture. A representative clone of human cells carrying the pDEL2 mini-gene $(C)$ demonstrates diminished extracellular deposition of fibrillin and grossly abnormal microfibrillar architecture. A control clone of mouse 3T3 cells transfected with the parent pcDNA3 expression vector $(D)$ demonstrates a normal cellular phenotype. A representative clone of pDEL2-harboring 3T3 cells is shown in $E$ and exhibits a dramatic reduction in fibrillin deposition, with only patches of disorganized microfibrils evident. An anti-fibronectin stain of 3T3 cells transfected with pDEL2 is shown in $F$ and demonstrates abundant and organized fibronectin deposition in a pattern identical to that of control cells (not shown). This verifies that the abnormalities observed in this study are not a reflection of global disruption of protein synthesis and processing secondary to transfection and/or neomycin selection. Untransfected cells plated in medium from atop cells carrying pDEL2 produced a pattern virtually identical to that seen in $E$ (not shown). Cells plated in medium from atop pcDNA3-harboring (control) cells produced the normal pattern of fibrillin deposition as in $D$ (not shown). This indicates that the mutant polypeptide is indeed secreted from the pDEL2-carrying cells, and its presence in culture medium can disrupt microfibrillar assembly. Aoyama et al. had previously failed to observe a dominant-negative effect when medium from atop Marfan fibroblast cell lines was added to control lines after the cells had already reached hyperconfluence (16). Taken together, these data suggest that mutant fibrillin monomer has the ability to impair matrix utilization of wild-type fibrillin but that the mutant protein has a diminished or absent capacity to interact with wild-type monomers that have already incorporated into microfibrils.

Pulse-chase analysis. Polyacrylamide electrophoresis gels of cellular protein extracts after ${ }^{35} \mathrm{~S}$-cysteine pulse and unlabeled-cysteine chase were evaluated by autoradiography and scanning densitometry as previously described (15). Calculated levels of fibrillin synthesis and deposition are presented in Table II. As would be expected from cells expressing two normal fibrillin alleles, $\sim 100 \%$ synthesis of fibrillin is observed in all of the pDEL2-transfected cell lines. Matrix deposition, however, is greatly reduced in these lines, to $50 \%$ or less of that seen in control lines. Data obtained from cells of patient MS8 are presented for reference (16). In these cells with one wildtype fibrillin allele, $\sim 50 \%$ fibrillin synthesis is observed, as expected.

These data suggest that expression of this mutant, truncated fibrillin polypeptide, even on a background of two wild-type fibrillin alleles, is sufficient to disrupt normal fibrillin aggregation and reproduce the MFS cellular phenotype.

\section{Discussion}

The hypothesis that MFS follows a dominant-negative mechanism of pathogenesis has been borne from several observations. The disease is inherited in an autosomal dominant fashion and is caused by mutations in fibrillin, a matrix protein known to multimerize. The severe deficiency of extracellular fibrillin, which is characteristic of classic MFS, is far below the amount predicted by a single functioning FBN1 allele. Lastly, very reduced levels of mutant transcript have been associated with extremely mild disease in patients. We now establish a dominant-negative effect in a cell culture model using a disease allele from a patient with severe MFS. We have shown that the 

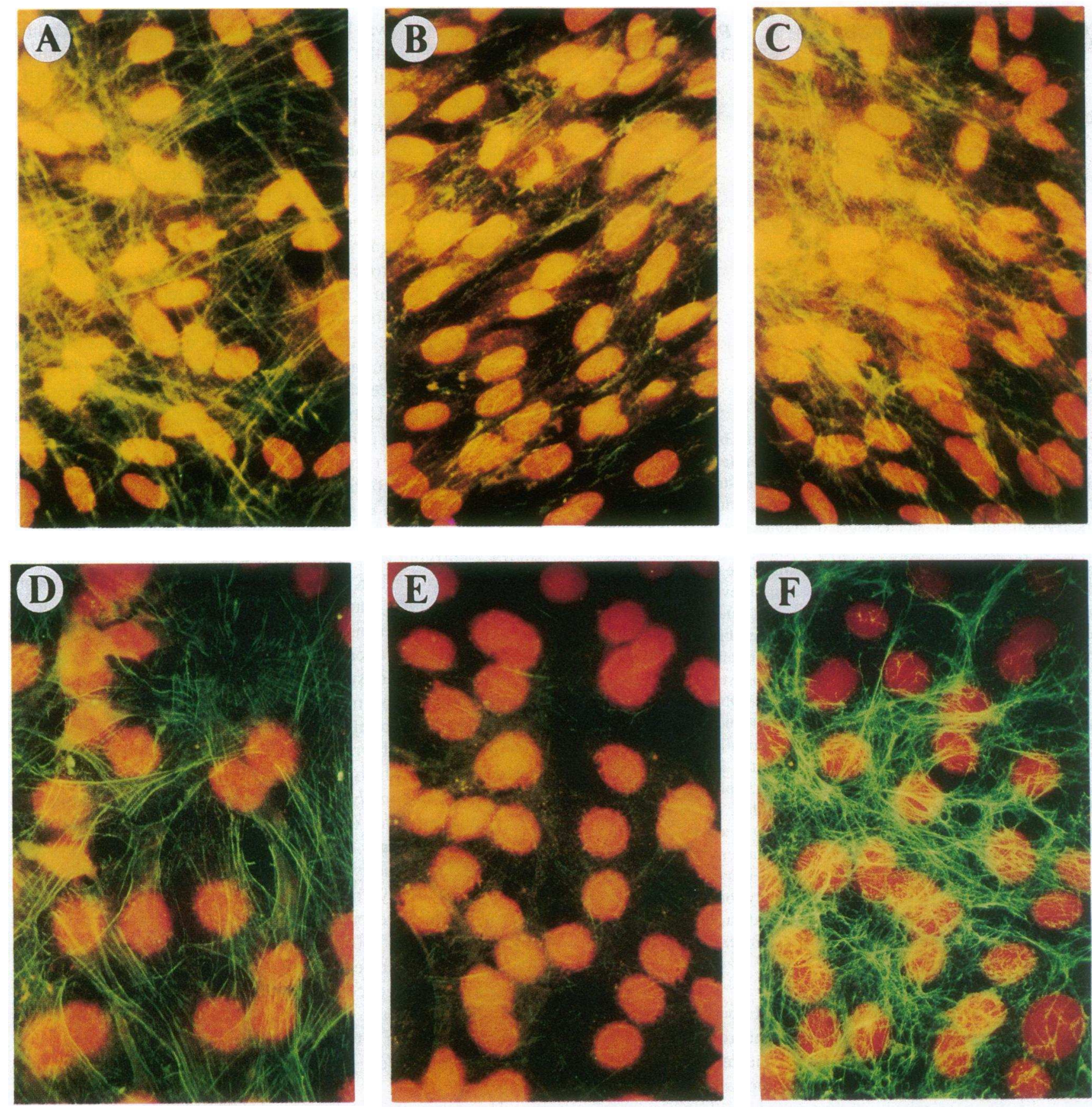

Figure 4. Photomicrographs of fibroblasts after indirect immunofluorescence with a monoclonal antibody to fibrillin $(A-E)$ and fibronectin $(F)$. Nuclei are stained with propidium iodide and appear orange. Microfibrils appear green. $(A)$ normal human fibroblasts; $(B)$ primary culture from patient MS-8; $(C)$ human fibroblasts transfected with pDEL2; $(D)$ 3T3 cells transfected with the control, insert-free pcDNA3 vector; $(E)$ 3T3 cells transfected with pDEL2; $(F)$ anti-fibronectin stain of 3T3 cells carrying pDEL2.

presence of a 100-amino acid polypeptide from the aminoterminus of fibrillin and encoded by this patient's mutant allele (see Fig. 1) is sufficient to perturb normal fibrillin multimer assembly and reproduce the MFS cellular phenotype, even on a background of two normal FBN1 alleles. These data further support the conclusion that deficiency of wild-type fibrillin is not the sole determinant of pathology in MFS, but rather, that some adverse, dominant-negative interaction between mutant and wild-type fibrillin monomers is responsible for microfibrillar disruption and consequent disease.

Correlating genotype with phenotype in MFS is an active and ongoing effort by numerous investigators. Among the patterns that have emerged from such studies is the observation that FBN1 missense mutations are associated with classic and severe MFS whereas mutations that produce premature termination codons and low levels of mutant transcript are associated with a range of phenotypic severity (17). The identification of one such mutation, a 4-bp insertion (5138insTTCA) found in a patient with extremely mild disease, served as a forerunner to the studies presented in this manuscript. This mutation produces a frameshift and consequent downstream premature termination codon. It has been well documented that premature 
Table II. Profile of Fibrillin Synthesis and Deposition into the Extracellular Matrix in Cells Transfected with pDEL2

\begin{tabular}{|c|c|c|c|c|}
\hline \multirow[t]{2}{*}{ Cell line } & & Synthesis & \multicolumn{2}{|c|}{ Deposition } \\
\hline & & $\% *$ & \multicolumn{2}{|c|}{$\% *$} \\
\hline Patient MS- $8^{\ddagger}$ & & 50.7 & \multicolumn{2}{|c|}{25.1} \\
\hline \multirow[t]{2}{*}{ Human } & clone 1 & 114.0 & \multicolumn{2}{|c|}{27.6} \\
\hline & & & $8 \mathrm{~h}$ & $20 \mathrm{~h}$ \\
\hline \multirow[t]{4}{*}{ Mouse 3T3 } & clone $\mathrm{E} 1$ & 100.9 & 46.7 & 51.6 \\
\hline & clone $\mathrm{E} 1$ & 113.8 & 36.4 & 32.1 \\
\hline & clone E3 & 106.3 & 38.6 & 39.4 \\
\hline & clone $\mathrm{E} 4$ & 101.4 & 40.8 & 40.5 \\
\hline
\end{tabular}

* Control cell lines were used to establish reference values for fibrillin synthesis and deposition. Sample loading was controlled as previously described $(15,16)$. ${ }^{\ddagger}$ Untransfected primary culture. Data per reference 16.

termination codons occurring before the penultimate exon of a gene are associated with reduced levels of mutant transcript $(21,22)$. In this patient mutant transcript was quantified to be $6 \%$ that of wild-type. The phenotype was restricted to mitral valve prolapse, long-bone overgrowth, early myopia, and striae distensae (17). This presentation does not meet current diagnostic criteria for MFS but would more appropriately be classified as the MASS (mitral, aortic, skin, skeletal) phenotype (23).

Two models have been proposed to reconcile the disparity in clinical presentation between that of this patient and those with FBN1 missense mutations and severe MFS (17). Briefly, the relative excess of wild-type compared to mutant fibrillin monomer may lead to a preponderance of normal multimer and therefore mild disease. Alternatively, the truncated polypeptide produced by the mutant allele may have a diminished or absent ability to interact with the wild-type fibrillin product; normal fibrillin multimers would still be generated, though perhaps in reduced amounts, resulting in a mild phenotype. The data put forth in this manuscript favor the first model. Our studies suggest that a disruptive interaction between mutant and wild-type fibrillin monomers indeed occurs and may be the basis for pathology in MFS. Increased relative amounts of wild-type monomer would produce a relative abundance of wild-type multimer and might therefore result in a mild phenotype.

Further insight has been gained from two other patients who harbored premature termination codon-producing mutations. Interestingly, mutant transcript levels in these individuals were 16 and $25 \%$, and their disease presentation was classic and severe (17). This observation of increased disease severity with increased mutant transcript level led us to augment the dominant-negative concept with the hypothesis that a threshold level of mutant transcript and therefore monomer, perhaps between 6 and $16 \%$, may be necessary to produce classic and severe disease. An analogous threshold hypothesis has been suggested for another connective tissue disorder, osteogenesis imperfecta (24). Again, the data presented in this manuscript are consistent with such a hypothesis. The del exon 2 allele was derived from the patient with $16 \%$ mutant transcript, and the immunohistochemical and pulse-chase profiles obtained from cells transfected with this allele closely resemble those obtained from primary cultures of this patient's fibroblasts (16). The data suggest that the level of expression of the mutant polypeptide in this study surpassed such a threshold and disrupted the normal multimerization of monomers produced from two intact FBN1 alleles.

Given this paradigm, the dominant-negative effect presented here may be the consequence of one or perhaps several molecular mechanisms. It is already known that fibrillin monomers aggregate in a head-to-tail fashion and have extensive intermolecular interactions principally mediated by disulfide bonds $(8$, $10,13)$. The present study suggests that the fibrillin aminoterminus is integral to this assembly process and that the 100amino acid polypeptide we have employed is capable of dominant-negative interaction with wild-type fibrillin monomers. Insight into how such an apparently mechanical derangement of normal microfibrillar assembly leads to the features observed in MFS (i.e., reduced extracellular fibrillin deposition and grossly abnormal microfibrillar architecture) perhaps can be gained from the characteristics of the fibrillin monomer itself. The bulk of the fibrillin backbone consists of EGF-like domains, which are motifs that have been found in a wide assortment of proteins with diverse biological functions (25). In fibrillin, 43 of 46 EGF-like repeats satisfy the consensus sequence for calcium binding, an event which is thought to stabilize protein structure, to promote protein-protein interactions, and to confer resistance to proteolysis $(17,26,27)$. Interestingly, the vast majority of FBN1 missense mutations in MFS patients occur within EGFlike domains and alter residues with putative significance for calcium binding $(17,28)$. In addition, in preparations of microfibrils in vitro, calcium removal has been shown to result in dramatically disrupted microfibrillar assembly (29). The similar cellular phenotype observed in this study may have a related basis. Perturbation of macromolecular structure may be the common factor.

One could envision many pathways by which the dominantnegative potential of mutant monomer could relate to disease pathogenesis. First, the abnormal phasing of fibrillin monomers during microfibrillogenesis induced by the mutant polypeptide may predispose to local environmental insults, such as proteolysis, with consequent impairment of microfibrillar architecture and hence functional integrity. Second, the identical scenario might predict instability and subsequent degradation of abnormal microfibrillar assemblies, leading to a gross deficiency of the entire extracellular fibrillin pool. Finally, mutant fibrillin transcript and/or monomer might interfere with the trafficking or intracellular processing of their wild-type counterparts. These mechanisms are not mutually exclusive and, indeed, may have a cumulative effect. Selected mechanisms may also be specific to certain mutant genotypes.

These and related issues will likely be resolved as further studies on the biology of normal and mutant fibrillin are undertaken. The cell culture system presented here may be employed in such investigations. For example, in the continuing effort to correlate genotype with phenotype in MFS, the system may be employed to interrogate the functional significance of various protein regions within fibrillin. The carboxy terminus and isolated central domains, when expressed in isolation, may be examined for their ability to impair normal microfibril formation. In addition, upon development of a full-length fibrillin expression construct, this system may be used to characterize effects of the growing number of identified disease-producing FBN1 missense mutations.

Another consequence of the findings presented here is their immediate bearing on the creation of an animal model of MFS. A complete understanding of the pathogenesis of this disease 
is likely to be attained only through study of transgenic models that mimic the physiologic complexity of the human system. Ideally, an animal model of MFS should provide an experimental paradigm that is applicable to human physiology and that enables diagnostic and therapeutic intervention. It would therefore facilitate the development, evaluation, and refinement of medical and surgical therapies for MFS and thus allow advances to be directly relevant to the human condition. Our data suggest that expression of the human extreme $5^{\prime}$ fibrillin-coding sequence may be sufficient, in isolation, to produce such a model. In this context, it should be noted that FBN1 is highly conserved across species. The porcine fibrillin cDNA, for example, has 92\% sequence identity to human FBN1 and $98 \%$ identity and 99\% homology at the amino acid level (Z. A. Eldadah and H. C. Dietz, unpublished data). This extreme conservation across the full 10-kb length of coding sequence suggests a functional significance to each of the individual domains within fibrillin. It also suggests that fibrillin monomers from one species may be able to interact with those of another. Indeed, in this study truncated human fibrillin polypeptides induced similar effects on human as well as murine microfibrils.

Ultimately, a principal objective of the fuller understanding of MFS gained though development of an animal model and other routes of investigation is relieving the clinical burden of patients. Toward this aim, verification of the dominant-negative nature of MFS pathogenesis now offers the possibility of mutant allele knockout as a potential strategy for gene therapy. Approaches based on antisense inhibition of mutant transcript expression, for example, may hold promise and can be tested in a cell culture system.

\section{Acknowledgments}

The authors wish to thank $\mathrm{L}$. Sakai for providing the anti-fibrillin monoclonal antibody used in this study, J. Bonadio for providing mouse fibrillin cDNA sequence prior to publication, and T. Aoyama for performing the pulse-chase analysis of the human cell lines.

This work was supported by grants from the National Institutes of Health (Z. A. Eldadah, H. C. Dietz), the Deutsche Forschungsgemeinschaft (T. Brenn), the National Marfan Foundation (H. Furthmayr, H. C. Dietz), and the Smilow Family Foundation (H. C. Dietz). Dr. Dietz is a Ross Research Scholar.

\section{References}

1. McKusick, V. A. 1972. Heritable Disorders of Connective Tissue. 4th ed. CV Mosby, St. Louis, MO. 61-223.

2. Pyeritz, R. E., and V. A. McKusick. 1979. The Marfan syndrome: diagnosis and management. N. Engl. J. Med. 300:772-777.

3. Pyeritz, R. E. 1990. Marfan syndrome. In Principles and Practice of Medica Genetics. Second edition. A. E. H. Emery and D. L. Rimoin, editors. ChurchillLivingstone, Inc., New York. 1047-1063.

4. Kainulainen, K., L. Pulkkinen, A. Savolainen, I. Kaitila, and L. Peltonen. 1990. Location on chromosome 15 of the gene defect causing Marfan syndrome. N. Engl. J. Med. 323:935-939.

5. Dietz, H. C., G. R. Cutting, R. E. Pyeritz, C. L. Maslen, L. Y. Sakai, G. M Corson, E. G. Puffenberger, A. Hamosh, E. Nanthakumar, S. Curristin, et al. 1991. Marfan syndrome caused by a recurrent de novo missense mutation in the fibrillin gene. Nature (Lond.). 352:337-339.

6. Lee, B., M. Godfrey, E. Vitale, H. Hori, M.-G. Mattei, M. Sarfarazi, P Tsipouras, F. Ramirez, and D. W. Hollister. 1991. Linkage of Marfan syndrome and a phenotypically related disorder to two different fibrillin genes. Nature (Lond.). 352:330-334.

7. Sakai, L. Y., D. R. Keene, and E. Engvall. 1986. Fibrillin, a new 350-kD glycoprotein, is a component of extracellular microfibrils. J. Cell Biol. 103:24992509.

8. Sakai, L. Y., D. R. Keene, R. W. Glanville, and H. P. Bachinger. 1991 Purification and partial characterization of fibrillin, a cysteine-rich structural component of connective tissue microfibrils. J. Biol. Chem. 266:14763-14770.

9. Maslen, C. L., G. M. Corson, B. K. Maddox, R. W. Glanville, and L. Y. Sakai. 1991. Partial sequence of a candidate gene for the Marfan syndrome Nature (Lond.). 352:334-337.

10. Corson, G. M., S. C. Chalberg, H. C. Dietz, N. L. Charbonneau, and L. Y. Sakai. 1993. Fibrillin binds calcium and is encoded by cDNAs that reveal a multidomain structure and alternatively spliced exons at the $5^{\prime}$ end. Genomics. 17:476-484.

11. Ramirez, F., L. Pereira, H. Zhang, and B. Lee. 1993. The fibrillin-Marfan syndrome connection. Bioessays. 15:589-594.

12. Milewicz, D. M., R. E. Pyeritz, E. S. Crawford, and P. H. Byers. 1992. Marfan syndrome: defective synthesis, secretion and extracellular matrix formation of fibrillin by cultured dermal fibroblasts. J. Clin. Invest. 89:79-86.

13. Keene, D. R., B. K. Maddox, H. J. Kuo, L. Y. Sakai, and R. W. Glanville. 1991. Extraction of extendable beaded structures and their identification as fibrillin-containing extracellular matrix microfibrils. J. Histochem. Cytochem. 39:441 449.

14. Maddox, B. K., L. Y. Sakai, D. R. Keene, and R. W. Glanville. 1991 Connective tissue microfibrils: isolation and characterization of three large pepsinresistant domains of fibrillin. J. Biol. Chem. 264:21381-21385.

15. Aoyama, T., K. Tynan, H. C. Dietz, U. Francke, and H. Furthmayr. 1993. Missense mutations impair intracellular processing of fibrillin and microfibril assembly in Marfan syndrome. Hum. Mol. Gen. 2:2135-2140.

16. Aoyama, T., U. Francke, H. C. Dietz, and H. Furthmayr. 1994. Quantitative differences in biosynthesis and extracellular deposition of fibrillin in cultured fibroblasts distinguish five groups of Marfan syndrome patients and suggest distinct pathogenic mechanisms. J. Clin. Invest. 94:130-137.

17. Dietz, H. C., I. McIntosh, L. Y. Sakai, G. M. Corson, S. C. Chalberg, R. E. Pyeritz, and C. A. Francomano. 1993. Four novel FBN1 mutations: significance for mutant transcript level and EGF-like domain calcium binding in the pathogenesis of Marfan syndrome. Genomics. 17:468-475.

18. Hollister, D. W., M. Godfrey, L. Y. Sakai, and R. E. Pyeritz. 1990 Immunohistologic abnormalities of the microfibrillar-fiber system in the Marfan syndrome. N. Engl. J. Med. 323:152-159.

19. Southern, E. M. 1975. Detection of specific sequences among DNA fragments separated by gel electrophoresis. J. Mol. Biol. 98:503-517.

20. Dietz, H. C., R. E. Pyeritz, E. G. Puffenberger, R. J. Kendzior, G. M Corson, C. L. Maslen, L. Y. Sakai, and C. A. Francomano. 1992. Marfan phenotype variability in a family segregating a missense mutation in the epiderma growth factor-like motif of the fibrillin gene. J. Clin. Invest. 89:1674-1680.

21. Urlaub, G., P. J. Mitchell, C. J. Ciudad, and L. A. Chasin. 1989. Nonsense mutations in the dihydrofolate reductase gene affect RNA processing. Mol. Cell. Biol. 9:2868-2880.

22. Cheng, J., M. Fogel-Petrovic, and L. E. Maquat. 1990. Translation to nea the distal end of the penultimate exon is required for normal levels of spliced triosephosphate isomerase mRNA. Mol. Cell. Biol. 10:5215-5225.

23. Glesby, M. J., and R. E. Pyeritz. 1989. Association of mitral valve prolapse and systemic abnormalities of connective tissue: a phenotypic continuum. J. Am. Med. Assoc. 262:523-528.

24. Prockop, D. J., A. Colige, H. Helminen, J. S. Khillan, R. Pereira, and P. Vandenberg. 1993. Mutations in type 1 procollagen that cause osteogenesis imperfecta: effects of the mutations on the assembly of collagen into fibrils, the basis of phenotypic variations, and potential antisense therapies. J. Bone Miner. Res. 2:S489-492.

25. Davis, C. G. 1990. The many faces of epidermal growth factor repeats New Biol. 2:410-419.

26. Cooke, R. M., A. J. Wikinson, M. Baron, A. Pastore, M. J. Tappin, I. D. Campbell, H. Gregory, and B. Sheard. 1987. The solution structure of human epidermal growth factor. Nature (Lond.). 327:339-341.

27. Handford, P. A., M. Mayhew, M. Baron, P. R. Winship, I. D. Campbell, and G. G. Brownlee. 1991. Key residues involved in calcium binding motifs in EGF-like domains. Nature (Lond.). 351:164-167.

28. Hewett, D. R., J. R. Lynch, R. Smith, and B. C. Sykes. 1993. A novel fibrillin mutation in the Marfan syndrome which could disrupt calcium binding of the epidermal growth factor-like module. Hum. Mol. Genet. 2:475-477.

29. Kielty, C. M., and C. A. Shuttleworth. 1993. The role of calcium in the organization of fibrillin microfibrils. FEBS (Fed. Eur. Biochem. Soc.). 336:323326. 